\title{
Hacia la observación de una sociedad venidera: Una entrevista con Dirk Baecker
}

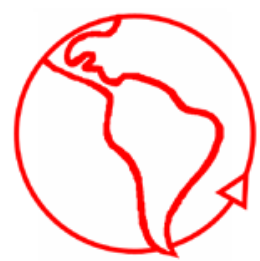

\author{
Towards Observation of a Next Society: An Interview with Dirk \\ Baecker
}

\section{Felipe Pérez- Solari}

Departamento de Historia, Universidad de Concepción, Chile

\section{Julio Labraña}

Departamento de Proyectos, Universidad Santa Federico Santa María, Chile

Resumen

Dirk Baecker ha sido reconocido como uno de los más connotados sociólogos alemanes con importantes trabajos en teoría de la cultura, teoría sociológica, sociología económica y sociología de las organizaciones, entre muchos otros. La siguiente entrevista inédita recorre diversos temas que han sido motivo de interés del sociólogo alemán como la teoría de sistemas sociales, los nuevos avances de la teoría sociológica, la sociedad mundial y sus importantes trabajos sobre lo que él ha llamado la 'sociedad venidera'.

Palabras Clave: Sociedad Venidera; Teoría de Sistemas Sociales, Teoría de Redes, Teoría Sociológica Contemporánea; Sociedad Mundial.

\begin{abstract}
Dirk Baecker has been recognized as one of the most prominent German sociologists with important works on cultural theory, sociological theory, economic sociology and organizational sociology, among others. The following unreleased interview covers various subjects of interest of the German sociologist, as social systems theory, new developments in sociological theory, world society and their important work on what he has called: the 'next society'.
\end{abstract}

Keywords: Next Society, Social Systems Theory, Networks Theory, Contemporary Sociological Theory; World Society.

\section{Introducción*}

La preocupación por el futuro de la sociedad ha marcado a la sociología desde sus orígenes. Baste pensar en Comte y la evolución del espíritu positivo, el diagnóstico de Marx sobre una resolución política de las contradicciones económicas, las consecuencias anómicas de la agudización de la división del trabajo en Durkheim o el despliegue de la "jaula de hierro" de racionalización y desencanto en Weber, entre otros muchos diagnósticos. La sociología contemporánea no es una excepción, observando con especial atención los características que pueden traer las siguientes décadas.

\footnotetext{
* Quisiéramos agradecer al Prof. Dr. Dirk Baecker el tiempo y la confianza al dar esta entrevista. De igual manera los invaluables aportes, críticas y comentarios de Fernando Robles, Kim Andersen y César Maríñez al manuscrito original de preguntas. Asimismo a Hugo Cadenas por su incansable y paciente guía en todo el proceso de elaboración y traducción de este texto. Cualquier error u omisión en la traducción es exclusiva responsabilidad de los autores.
} 
La teoría de sistemas sociales autopoiéticos no escapa a esta tendencia. El mismo Luhmann ha reflexionado brevemente sobre escenarios cuyos rasgos se comienzan a ver hoy en día, especialmente las consecuencias de una primacía de la distinción inclusión/exclusión. Fenómenos emergentes como la masificación del computador o la proliferación de redes en la sociedad contemporánea recibieron una menor atención por parte del sociólogo de Bielefeld, si bien han sido abordados desde una perspectiva sistémica por otros autores. Uno de ellos es el Prof. Dr. Dirk Baecker, destacado investigador de la Universidad Zeppelin de Alemania, que ha reflexionado sobre estos fenómenos. ¿Cómo es esta sociedad venidera, que comenzamos recién a experimentar? ¿con qué elementos es posible describirla sociológicamente? En las siguientes líneas abordamos estos y otros interesantes temas.

\section{Teoría de sistemas}

I) Profesor Baecker, usted es uno de los sociólogos sistémicos más importantes del mundo, con importantes investigaciones sobre Bancos, Análisis Cultural, Organizaciones, modalizaciones utilizando Cálculo de la Forma, management, entre otras. En su opinión, ¿qué resultados ha alcanzado la teoría de sistemas sociales en los últimos 40 años? ¿se encuentra en buena forma la herencia del Prof. Luhmann?

Desde que Talcott Parsons en conjunto con otros investigadores crearon la teoría de sistemas durante la década del cincuenta, esta siempre se ha encontrado en un estado más bien precario. Parsons persiguió un programa de integración disciplinaria muy fuerte entre sociología, psicología, antropología y economía en la Universidad de Harvard, para así desarrollar una gran teoría unificada para las ciencias sociales, un esfuerzo que recibió una fuerte resistencia en la década de los setentas debido a su postura supuestamente conservadora. De igual manera Luhmann en las décadas de los setentas, ochentas y noventas no vaciló en avanzar en una resuelta política de integración teórica en la sociología, una política que, de seguro, nunca dejó el nivel de los textos ni fue tan lejos como para desarrollar una escuela y de ese modo asegurarse que sus seguidores postulasen exitosamente a cátedras universitarias. Tanto Parsons como Luhmann fueron muy claros y decisivos en sostener que la noción de sistemas es una de las ideas más fructíferas en sociología, ya que era, y es, capaz de llamar la atención de los estudiosos de las operaciones sociales que tienen que ser capaces, al mismo tiempo, de hacer una diferencia y reproducirla en el tiempo. Sin embargo, su propio dominio de la investigación empírica, fue siempre altamente reflexivo en referencia a sistemas a ser investigados, fuesen estos interacciones, organizaciones, o un subsistema social, lo cual probó ser frustrante para cualquier colega que no fuese capaz de poseer un ingenio similar.

Por lo tanto, las razones ideológicas fueron muy útiles para rechazar de plano la teoría de sistemas sociales, para leer los textos de Parsons y Luhmann en el mejor de los casos con sospechas (pese a que había muchos círculos de lectura entre académicos de todas las disciplinas), nunca analizando su proyecto teórico de una forma realmente seria. Sin embargo, no hay motivo para la desesperación, pues siempre han sido unos pocos los sociólogos que persiguen un programa teórico en la sociología, y entre estos son incluso menos los que siguen el programa de la teoría de sistemas.

El estado de este tipo de pensamiento es, sin embargo, fuerte y es apoyado por dos tipos de intereses. Uno de tipo internacional, así como otro que desea mantener un modo de intercambio interdisciplinario entre sociología, por un lado, y ciencias cognitivas de diversas clases, principalmente cibernética, ciencias computacionales, matemáticas, biología, y neurofisiología, por otro. La herencia de Parsons y Luhmann parece encontrarse en mal estado ya que pocas escuelas siguen abiertamente sus programas, pero por el contrario, se ve que está en buena forma si se toma en cuenta que el interés por la operación Ilamada 'lo social' aparece aquí y allí, una y otra vez. Junto con la teoría 
de los juegos, el institucionalismo y la teoría de redes, la teoría de sistemas sociales es uno de los programas teóricos de la sociología que ciertamente estará con nosotros en el futuro.

II) En su opinión, ¿cuáles han sido los debates más importantes entre los pensadores sistémicos en los últimos años? ¿qué temas considera Ud. más relevantes en la teoría de sistemas?

Existen al menos tres temas que están siendo debatidos por pensadores sistémicos en sociología, y por supuesto en muchos otros campos diferentes. El primer tema es si acaso es todavía posible usar el concepto biológico de autopoiesis, inventado por Humberto Maturana, Francisco Varela y Ricardo Uribe, cuando es traducido en términos de una teoría de sistemas generales, tal como lo hizo Heinz von Foerster con la cibernética de segundo orden, y también a la sociología. El segundo tema se centra en la pregunta de si acaso resulta una buena idea apoyarse en el libro Laws of Form de George Spencer- Brown para avanzar en una teoría de sistemas sociales. El tercer tema, finalmente, es si acaso Luhmann con su chef d'œuvre final La sociedad de la sociedad estaba preparado para considerar, tanto una transformación de la modernidad en una sociedad venidera, debido a la introducción de la electricidad, el computador y la internet; así como para decirle adiós a la idea de diferenciación funcional como el principio rector de la diferenciación de una sociedad venidera. Todos estos tópicos son controversiales. Existen pensadores sistémicos en sociología que consideran el análisis funcional desarrollado por Luhmann en 1960 y 1970 como un estado del arte inigualable, hay otros que dudan de la utilidad de cualquier pensamiento en términos de clausura operacional, hay unos pocos que no están satisfechos con la introducción del observador por parte de Luhmann y casi nadie entra en la discusión acerca del uso del cálculo de indicaciones de Spencer-Brown. Por supuesto, existen buenas razones para todo esto, y en todos sus casos particulares.

III) Profesor Baecker, Ud. ha utilizado el Laws of Forms de George Spencer- Brown en sus obras Lenin's twist, Hitler Swarm, Form of the Game, A Calculus for Autopoiesis, entre otras. ¿Qué piensa acerca de la posibilidad de usar este cálculo en áreas de las ciencias sociales tales como la teoría social y la metodología?

Bueno, creo que el modo en el que uso el cálculo, este es realmente útil. El cálculo no sólo ofrece la posibilidad de resolver el problema planteado por la teoría de la autopoiesis respecto de cómo la clausura operativa es capaz de cerrar y abrir, en una sola operación, el sistema, sino que también es altamente apropiado para tomar en consideración una comprensión de la comunicación como referida siempre y al mismo tiempo, tanto al habla como al silencio, tanto al conocimiento como a la ignorancia. El único hecho concreto acerca de cualquier sistema social empírico es que existen estados no marcados corriendo paralelos a cualquier comunicación, temas que deben ser incluidos como excluidos. Ello demostraría que es casi imposible desarrollar métodos de investigación empírica que consideren estos estados no marcados y que realicen además los protocolos apropiados para codificarlos y documentarlos, si bien esto no puede impedir que la teoría desarrolle una noción de dicha característica decisiva de cualquier comunicación.

Si se agregan las ideas de Spencer-Brown respecto de cómo introducir al observador, la autorreferencia y la paradoja dentro de un cálculo de operaciones, se obtienen allí más razones para tomar seriamente en cuenta su idea acerca de una forma de la distinción. Es fascinante ver cómo Spencer-Brown, se encuentra de hecho - y presumiblemente sin saberlo- desarrollando algunas de las ideas más radicales del idealismo alemán, tales como la infinitud como presupuesto de cualquier operación que espera encontrar continuidad, o el vacío del sí- mismo al que refiere toda autorreferencia, ideas que sólo pueden ser entendidas correctamente si se entra en el pensamiento filosófico de la negación por parte de Hegel, Heidegger, o Gotthard Günther, y de este modo ver cómo un 
lado de la distinción es capaz de negar al mismo tiempo al otro lado, e implicarlo mediante una negación.

El entendimiento de la 'forma' de Spencer-Brown fue incluso intuido con fuerza por personas como el Conde de Shaftesbury, Wilhelm von Humboldt, Karl Marx y George Simmel. Harrison C. White escribió una vez (en la segunda edición de su libro Identidad y Control) que se encuentra seguro de perderle la pista al pensamiento sociológico, si se toma en cuenta el cálculo de la forma de Spencer-Brown. Creo que, por el contrario, el cálculo nos lleva de vuelta a las ideas más fascinantes acerca de lo social. No conozco acción o comunicación que no se encuentre oscilando entre síes o noes, o entre los costados de los dilemas descritos por las variables pautas de Parsons, en exactamente el mismo modo en que Spencer- Brown comprende las funciones de oscilación y memoria, en el importantísimo capítulo 11 de su Laws of Form.

IV) La posibilidad de una intervención social sistémica ha sido problemática para la teoría de sistemas sociales desarrollada por Niklas Luhmann, especialmente por sus conceptos de determinismo estructural y clausura operacional. Ello es evidenciado en el libro de Jürgen Habermas, Facticidad y validez, donde el autor discute los argumentos de Helmut Willke respecto de una coordinación organizacional experta entre sistemas sociales, acusándolo de no considerar adecuadamente el problema de la intersubjetividad. ¿Cómo percibe Ud. este debate? ¿Qué es lo que piensa acerca de una "teoría de la intervención" basada en las propuestas de Luhmann?

Desde mi punto de vista, una 'teoría de la intervención' sólo puede basarse en una teoría de los sistemas complejos, en el sentido de W. Ross Ashby. Lo anterior significa que nunca hay una comprensión de esta clase de sistemas ni tampoco una posibilidad de instruirlos desde el exterior. Lo que sucede, en cambio, es que los observadores entran en interacción con estos sistemas, ofreciendo observaciones, descripciones, oportunidades y restricciones que pueden o no provocar ciertas respuestas en los sistemas. Para esto Ashby utiliza el concepto de 'control', lo cual no implica dominación sino más bien memoria. Un observador en una relación con un sistema complejo (sea su familia, una organización, el sistema político, los mercados económicos, movimientos sociales u otro) controla lo que él o ella hace con el sistema, y el sistema lo que hace con él o ella, ya sea para repetir o modificar la siguiente acción respecto a la anterior. De esta manera, una memoria se construye relacionándose con ideas tanto sobre causalidad, como sobre improbabilidad, obstinación e inercia, que estructuran la interacción entre observador y sistemas dentro de la perspectiva del observador. Nadie sabe ni puede saber qué hace el sistema con esto. Cualquier conocimiento sobre el sistema es sólo otro intento de 'controlarlo' mediante la memoria que se tiene de las propias acciones y experiencias. En ningún punto entra en consideración la intersubjetividad. El término es sólo un intento para evitar aceptar la improbabilidad de la comunicación.

V) Dentro de las teorías sociológicas contemporáneas Ud. ha trabajado las conexiones entre la teoría de sistemas de Luhmann y la teoría de redes de Harrison White. En su opinión, ¿cómo pueden ambas teorías contribuir entre sí?

Primero que nada, es importante enfatizar que ambas teorías coinciden en desarrollar una complejidad teórica propia con miras a captar la complejidad social. Existen muchas buenas razones para mantenerlas separadas y desarrollarlas en paralelo. Sin embargo, dado que a la teoría de redes de White le falta una noción de operaciones temporales, y la teoría de sistemas de Luhmann es más bien pobre en describir las estructuras de cualquier sistema social, parece evidente que podría ser una buena idea intentar acercamientos entre ambas teorías para compensar las deficiencias de cada una. Resulta interesante observar que la noción de autopoiesis de Maturana, Varela y Uribe hace un 
marcado énfasis en el rol de la red que produce y es producida por los elementos de cualquier sistema operacionalmente cerrado. Luhmann, por su parte, se centró más bien en la clausura operativa que en las redes cuando generalizó y reespecificó el concepto para su uso en la teoría de sistemas sociales. Nos encontramos aquí posiblemente con el engranaje que hace posible que las ideas de integración puedan tener efecto. Por supuesto, se necesita la idea de la forma de una distinción para combinar la operación de los sistemas con su búsqueda de identidad y control dentro de una red.

VI) En su artículo, Gyspsi Reason: Niklas Luhmann's Sociological Enlightenment, Ud. comenta que extraña poder discutir con el Prof. Luhmann sobre los nuevos avances de la teoría. ¿Cómo se siente hoy respecto a esa opinión? ¿Considera que la teoría ha despegado por su propia cuenta?

No, creo que todavía él hace falta y continuará siendo así. Él era, por una parte, totalmente singular en su comprensión de la literatura empírica y, por otra parte, poseía la elegancia, parsimonia y precisión para inventar las ideas y nociones que dieron a la teoría su arquitectura coherente y su ámbito de aplicabilidad. No creo que necesitemos todavía su autoridad, aunque podría ayudar en algunas ocasiones, sino que necesitamos aún sus ideas simples de enorme alcance, las cuales mantenían a su teoría en marcha. Consideremos como ejemplo su recepción de la noción de autopoiesis, la que le da un giro temporal a la comprensión de los componentes o elementos. Esto hace de los eventos una noción básica en teoría sociológica, una noción aún en general ausente, salvo en la teoría de sistemas biológicos. Sin la noción de eventos no se podría hablar acerca de comunicación o sentido, como Luhmann lo hizo, o sobre la interconexión dentro y entre sistemas, como nos gustaría hacer a nosotros.

VII) Desde los debates que sostuvieron Luhmann y Habermas, la teoría de sistemas sociales ha sido acusada de tener un carácter políticamente conservador. ¿Cuál es su opinión a esta consideración?

Debo decir que me es difícil encontrar razones para preocuparme sobre esto, dado que como he dicho anteriormente, estas suposiciones han sido una justificación para rechazar el pensamiento de Parsons y de Luhmann. Muchos Marxistas lamentaron esta decisión dado que sabían que sólo una teoría de sistemas sociales podría ser capaz de desarrollar aún más la teoría de la sociedad capitalista. Seguro, tanto Parsons como Luhmann sabían bastante bien cuán infantiles eran todas las ideas acerca de revolucionar la sociedad y transformarla de un solo golpe en una mejor, y ambos preferían referirse a las diversas funciones de la sociedad que debían ser sostenidas cuando se intenta cambiar una sola institución, sólo para hacer que ese cambio tenga lugar. Cualquier sociólogo es conservador en este sentido, porque tiene el mayor respeto por cómo las personas de hecho son capaces de manejar las tareas de su vida diaria, profesional y familiar. Ningún sociólogo piensa que el conocimiento adquirido leyendo libros pueda ayudar a cambiar el más simple lugar de la sociedad. Pero todo esto, tanto en Parsons como en Luhmann, nunca significó que ellos no fueran capaces de describir concienzudamente cómo los sistemas al mantenerse a sí mismos eran perfectamente capaces de provocar estragos en el resto de esferas de la sociedad.

\section{Sociedad Mundial}

VIII) En América Latina la sociología ha sido tradicionalmente asociada a una función crítica y de transformación de la sociedad. ¿Qué rol brinda Ud. a la sociología en la sociedad contemporánea? ¿Es todavía posible creer que por medio de la sociología se podría transformar la sociedad? 
La sociología en estos momentos se encuentra ocupada actualizando su entendimiento y descripción de la sociedad contemporánea, así como progresando en sus herramientas teóricas y empíricas para llevar a cabo su misión. Como a Luhmann le gustaba decir, es trabajosa su propia transformación y debería atenerse a eso, lo que ya de hecho es una tarea enorme. Cualquier interesado en política, economía, educación, derecho, religión o arte con ganas de transformar esas esferas de la sociedad debe tener precaución de mirar qué es lo que la sociología está tratando de decir sobre la complejidad, autorganización y la institucionalización contingente de las funciones societales. Pero como sabe muy bien cualquier profesional que habla con otros profesionales, hablar y dar a tus compañeros todas las posibilidades para que te entiendan es una idea mucho mejor que tratar de influirles, educarles, enseñarles o transformarles de cualquier manera. ¿Quién eres tú para siquiera intentarlo? Los otros a los que les hablas puede que no sean el problema, sino la solución a un problema del que no puedes siquiera tener idea.

IX) Una discusión recurrente que gira en torno a las condiciones de la moderna sociedad latinoamericana refiere a la consecuencia de la radicalización de la apatía de los sistemas funcionales o sobre su desdiferenciación en manos de los sistemas político o económico. Profesor Baecker, ¿cómo observa Ud. este debate? ¿Desdiferenciación o radicalización de la indiferencia de los sistemas funcionales?

Yo observo una sociedad en red emergente, que está experimentando consigo misma al desarrollar estructuras inherentes a los subsistemas funcionales tan típicos de la sociedad moderna, los que a su vez son producto de la introducción de la imprenta. Dado que en estos días tenemos que lidiar con computadores, sus derivados y redes, es que observamos un proceso de desdiferenciación entre las funciones de la sociedad moderna 0 , en otras palabras, entre las maneras en que esas funciones fueron institucionalizadas.

Siempre me ha impresionado el hecho de que la gente que tiene la afición de hablar de desdiferenciación, use el mismo concepto de especificaciones funcionales, las que piensan que ya no serían útiles. Ellos hablan de política, economía, derecho, arte, religión, educación y otros, como esferas diferenciadas que deben ser tomadas en serio. De hecho, ¿cómo podríamos huir, ellos y nosotros, de esta manera de hablar? Según mi parecer, la indiferencia a la que Uds. hacen referencia es una correlación de redes híbridas constituyéndose a sí mismas mediante límites y distinciones modernas, las que están volviéndose más insensibles a influencias externas que lo que cualquier subsistema funcional jamás pudo.

Esas redes híbridas tienen su propia economía, su propio derecho, su propia política, su propia religión y su propia estética, se vuelven así apáticas -como ustedes señalan- a cruces referenciales que intenten forzarlas a tomar nota de lo que tienden a excluir. Esta es una sociedad venidera [next society], como la nombró Peter Drucker, experimentando con nuevas formas de diferenciación. Esto no significa que estemos siempre felices con estas formas. Sin embargo, todavía tenemos que desarrollar las herramientas apropiadas para describirlas y entender cómo funcionan.

X) Hablando sobre Europa, la actual crisis económica ha forzado a muchos gobiernos a confrontar y lidiar con la crisis mediante políticas de austeridad, nueva regulación de los bancos, etc. En otras palabras, no ha habido una propuesta uniforme por parte de la Unión Europea (v. gr. Grecia, España e Islandia). Profesor Baecker, ¿cómo observa usted la capacidad de los gobiernos para arreglar esta crisis económica? ¿es posible detener la crisis o ellas son más bien una constante?

Como ustedes saben, las crisis son la manera en que la sociedad se asegura de que todos sepan que ciertas cosas no van a continuar de la misma manera: son maneras de reproducir la sociedad. De allí que debo estar satisfecho con que los países europeos descubrieran el modo de reproducción de la crisis y que hayan intentado cambiar lo que 
ellos mismos inventaron antes. La introducción del Euro sobrecargó las habilidades de los países para encontrar sus propios modos de desarrollar tanto la economía como la política. El cambio de moneda los forzó a un acoplamiento estricto entre sus políticas, mientras que los acoplamientos flojos habrían sido necesarios. Los gobiernos europeos, de hecho, están atareados con la crisis, y estoy seguro que manejarán la situación siempre que mantengan un ojo puesto en políticas alternativas. Estos gobiernos actualmente son expertos en retrasar exitosamente todo tipo de respuestas sobre las crisis que son inciertas. En referencia a esto Europa hizo algún progreso si comparamos situaciones de hace setenta u ochenta años, donde mucha gente tenía que resolver las crisis de forma muy rápida y de muy mala manera.

\section{Sociología en el Siglo XX}

XI) Hoy en día el concepto de cultura posee una posición bastante problemática en las ciencias sociales. Sin embargo Ud. en su artículo Meaning of Culture tiene otra dimensión sobre la utilidad del concepto para el análisis sociológico. Dado lo anterior ¿cómo observa este debate y la utilidad de este concepto?

La cultura, desde que Jean Jacques Rousseau inventó la crítica cultural, ofrece la posibilidad de relacionar la estructura social con la sensibilidad humana. No necesitamos preguntarnos, como Rousseau, qué sociedad vuelve a los humanos más felices o infelices, pero aún podemos preguntarnos por las relaciones entre cuerpo, mente y sociedad que la sociología clásica, en conjunto con las versiones más recientes, han a menudo descuidado. Tan pronto se sigue a Parsons o Luhmann y se considera la forma de la acción o de la comunicación, como una tipología que rodea los tres aspectos incompatibles de lo psíquico, lo orgánico y de los sistemas sociales, se necesita de un concepto que actúe como un comodín al separar y enlazar esos sistemas. Bronislaw Malinowski en su Una teoría científica de la cultura comprende a la cultura como un mecanismo de balanceo entre las actividades humanas y las necesidades, entre estructuras sociales y entornos. Podemos tomar esa idea y desarrollarla para posteriormente complementarla con los actuales desarrollos de las ciencias cognitivas, las que han tratado en vano hasta hoy de convertirse en multirreferenciales, como deberían ser, para dar cuenta de la complejidad de la condición humana. Sumado a lo anterior, la cultura no consiste solamente en la mantención de tradiciones, cultivo de la conducta, disciplinamiento del habla y la acción, sino también en ser siempre crítica de los estados contemporáneos de la sociedad, de modo que se termina con un concepto que puede no tener prácticamente interrelaciones teóricas y entrelazados de lenguajes positivo/negativos de descripciones de la sociedad. En mi caso tomo el pronunciamiento de disenso de la cultura con la sociedad dentro de la sociedad, y miro a las preguntas sobre la felicidad y la infelicidad o del cuerpo, la mente y lo social para razonar tanto sobre las afirmaciones como las críticas. Muchos escépticos del concepto de cultura enfatizan su incurable difusividad, pero por mi parte pienso que dentro de esa cualidad hay una muy aguda calculadora que está actualmente funcionando.

XII) Profesor Baecker ¿Qué teorías sociológicas considera más relevantes actualmente? Anteriormente mencionamos su trabajo con la teoría de redes, en este contexto, ¿piensa en una posible congruencia de una gran teoría (redes y sistemas) que se enfoque en los procesos emergentes de la sociedad? o por el contrario, ¿cree que las diferencias esenciales entre ellas deben ser mantenidas?

De alguna manera he intentado contestar esta pregunta previamente. Pienso que hay buenas razones para mantener las teorías separadas, no olvidando que existen todavía modelos económicos muy atractivos o también modelos psicoanalíticos y psicoterapéuticos, así como narraciones históricas que compiten vigorosamente con el pensamiento sociológico. Una gran teoría social unificada no es un tema para pensar 
sentado en un sillón. Piensen solamente en el destino del pensamiento evolucionista que siempre está abarcando una integración del tiempo, estructura y variación, no obstante no se ha convertido en una teoría cognitiva unificada. En cierto sentido yo prefiero mirar, incluso a nuestras poderosas y grandes teorías, brillantes como son la teoría de juegos de Jon Elster, la teoría de redes de Harrison C. White y la teoría de sistemas de Luhmann, como teorías de alcance medio en el clásico sentido de Merton, como embarcaderos evolutivos [evolutionary jetties] ${ }^{1}$ como le gustaba decir a Derrida o una meseta en la filosofía en términos de Deleuze, que combine esas ideas teóricas con aquellas preguntas empíricas. Mirémoslas desmoronarse de nuevo e intentemos otra cosa pues ¿qué haríamos si un consenso teórico fuese alcanzado?

\section{El futuro de la sociedad}

XIII) Profesor Baecker, Ud. ha explorado las consecuencias de las nuevas tecnologías como el internet en la sociedad contemporánea. ¿Puede explicarnos qué diferencias introduce esta sociedad venidera? ¿una sociedad que tiene el computador como medio es opuesta a la conceptualización tradicional de la modernidad?

Si el computador y sus redes es considerado como una sobrecarga para la sociedad contemporánea, como lo hizo la imprenta en la sociedad tradicional cuando libros, artículos, archivos, papel moneda, certificados de escuela, etc., fueron extensamente usados, entonces en efecto es mejor que comencemos a observar nuevas culturas y estructuras de una emergente sociedad venidera. Si su estructura de diferenciación son las redes y no los subsistemas funcionales; si su cultura no consiste más en creer en un equilibrio racional sino en apreciar las turbulencias fractales y los argumento nítidos sobre los códigos de la máquina que distingue entre la carne y los seres humanos y si los negocios, la política, sectas, grupos de arte e incluso las universidades pueden ser consideradas proyectos de control altamente precarios y no organizaciones racionales dentro de subsistemas funcionales, entonces icómo podemos imaginarnos esa sociedad venidera? ¿No estamos mirando ya estas estructuras sociales aún vivas en el así llamado tercer mundo, el que fue considerado condenado porque tradicionalmente ayer y hoy era un ejemplo de cómo lidiar con la indeterminación, la incalculabilidad, el riesgo y el peligro? El computador es tanto un desafío a la vida moderna, porque nos entrega una conectividad nunca antes vista, como una herramienta para lidiar con dicho desafío de maneras imprevisibles. Deberíamos asegurarnos solamente que esta herramienta esté en las manos de la mayor cantidad de personas posibles, sin excluir posibilidades de encontrar con su ayuda nuevas posibilidades de autorganización.

XIV) La relevancia de redes sociales como Twitter, Facebook y otras crece cada día. En estos medios es posible observar la generación de una forma alternativa de información, así como nuevos movimientos de protesta como Anonymous, que no dejan de lado la interacción pero la desacoplan de la presencia física. ¿Cómo observa estas tecnologías? ¿Cómo consideraría las nuevas demandas transmitidas a la gente? ¿Cuál piensa que será su desarrollo en el futuro de la sociedad venidera?

En realidad no tengo idea. El futuro es desconocido y, hasta donde sabemos, seguirá así. No hago una previsión, pero me gusta hablar sobre nuestra gran ignorancia del futuro, pues es la razón más importante para que la sociedad mundial todavía sea capaz de

\footnotetext{
${ }^{1}$ Traducido del concepto evolutionary jetties definido por Derrida como "the force of that movement which is no subject, project, or object, not even rejection, but in which takes place any production and any determination, which finds its possibility in the jetty". Derrida, Jacques (1990) Some Statements and Truisms about Neologisms, Newisms, Postisms, Parasitisms, and Other Small Seismisms, pp. 63-94 in David Carroll (ed.) The States of 'Theory': History, Art, and Critical Discourse. New York: Columbia University Press.
} 
integrarse. No sabemos qué es lo que está pasando con las redes sociales, es por ello que las estamos observando. Lo mismo sucede con la economía, la política, la religión, el amor o la ciencia. Los inversionistas chequean el mercado porque no saben ni cómo ni cuándo sus clientes aparecerán. Los políticos tienen todavía un ojo puesto en los votantes y sus competidores para estar seguros, pues no saben si serán electos de nuevo. El creyente trata de llevar una vida piadosa porque no sabe si llegará o no al reino de los cielos. De igual manera quien ama está atento porque no sabe cuánto tiempo más será amado o amada. Alabemos nuestra ignorancia sobre el futuro.

XV) Profesor Baecker, en Zukunftsfähigkeit: 16 Thesen zur nächsten Gesellschaft ${ }^{2}$ menciona que la estructura de la sociedad venidera, no sería la diferenciación funcional sino las redes. ¿Puede explicar las consecuencias de esto para la forma de la evolución? ¿en qué sentido la sociedad ya no será la misma?

Bien, creo que ya hablamos algo de esto. Conforme las nuevas estructuras emergen y apenas logramos entenderlas, observamos a mucha gente lidiando con todo tipo de recursos. Observando estas estructuras comenzamos a desarrollar una terminología útil tanto para la sociología como para la sociedad. Ese lenguaje es debatido entre los científicos, activistas, intelectuales y muchos otros. Debemos estar atentos a ese lenguaje e incluso tener cuidado de evitar hablar todos el mismo tipo de Globish ${ }^{3}$ o inglés simple al que nos hemos acostumbrado cada vez más, ¿no hay ningún lenguaje local o sintaxis vernácula, semántica o cálculo pragmático en todo esto? La transformación de la modernidad en una sociedad venidera posiblemente está ocurriendo, debemos mantenernos seguros de que podemos hablar la mayor cantidad de lenguas para ampliar lo más que podamos las posibilidades de la evolución en las cuales se basa la sociedad e instituir varias maneras para no entender y de igual manera malinterpretarnos entre nosotros, tanto como sea posible.

XVI) Profesor Baecker, muchas gracias por su tiempo. ¿Quisiera mandar un mensaje a los investigadores sistémicos en América Latina?

Sigan trabajando, manténganse alertas y cuídense. De igual manera, muchas gracias a ambos por sus interesantes preguntas. RM

\section{Sobre los autores}

Felipe Pérez-Solari es historiador de la Universidad de Concepción, Chile. Actualmente se desempeña como investigador en el Departamento de Historia de la Universidad de Concepción, Chile y como becario Conicyt, Chile. Sus áreas de especialización son: Teoría de Sistemas Sociales, sociología política, sociología histórica, teoría de la historia, historia moderna y contemporánea.

fperezsolari@ug.uchile.cl

Julio Labraña es sociólogo de la Universidad de Concepción, Chile. Actualmente se desempeña como investigador en la Universidad Federico Santa María- Sede Concepción. Sus áreas de especialización son: Teoría sociológica, teoría de sistemas sociales, sociología de la educación, metodologías de la investigación social, políticas de intervención, desigualdad social.

jlabrana@ug.uchile.cl

\footnotetext{
${ }^{2}$ Sustentabilidad: 16 Tesis sobre la sociedad venidera publicado en: Revue für politisches Management, Cuaderno 9 (2011), pp. 9-11. Véase sobre el contexto: Dirk Baecker (2007). Studien zur nächsten Gesellschaft, Suhrkamp, Frankfurt a. M. Traducción de Fernando Robles.

${ }^{3}$ Neologismo inventado por Jean Paule-Nerriere, ex presidente de IBM, desde las palabras Global e English para dar cuenta del manejo del idioma inglés con sólo mil quinientas palabras.
} 
Contacto

Universidad Técnica Federico Santa María- Sede Concepción

Alemparte 943 - Hualpén

Chile

Recibido: Junio 2013

Aceptado: Agosto 2013 